Supporting Information for:

\title{
Ionic Organic Microcrystals with Bright Deep-Blue Luminescence and Acid/Base Vapor Response
}

Zhong-Qiu Li ${ }^{\dagger,+, \# ~ J i a n-H o n g ~ T a n g, ~}{ }^{\dagger, \#}$ Yan-Qin He, ${ }^{\dagger, \S}$ Zhong-Liang Gong, ${ }^{\dagger}$ Jiang-Yang Shao, ${ }^{\dagger}$ and Yu-Wu Zhong*,t,

${ }^{\dagger}$ Beijing National Laboratory for Molecular Sciences, CAS Key Laboratory of Photochemistry, CAS Research/Education Center for Excellence in Molecular Sciences, Institute of Chemistry, Chinese Academy of Sciences, Beijing 100190, China

${ }^{\$}$ School of Chemical Sciences, University of Chinese Academy of Sciences, Beijing 100049, China

${ }^{\S}$ Institute of BioPharmaceutical Research, Liaocheng University, 1 Hunan Road, Liaocheng, Shandong 252000, China

\#These authors contribute equally to this work.

Corresponding Author

*zhongyuwu@iccas.ac.cn (Y.-W.Z.) 

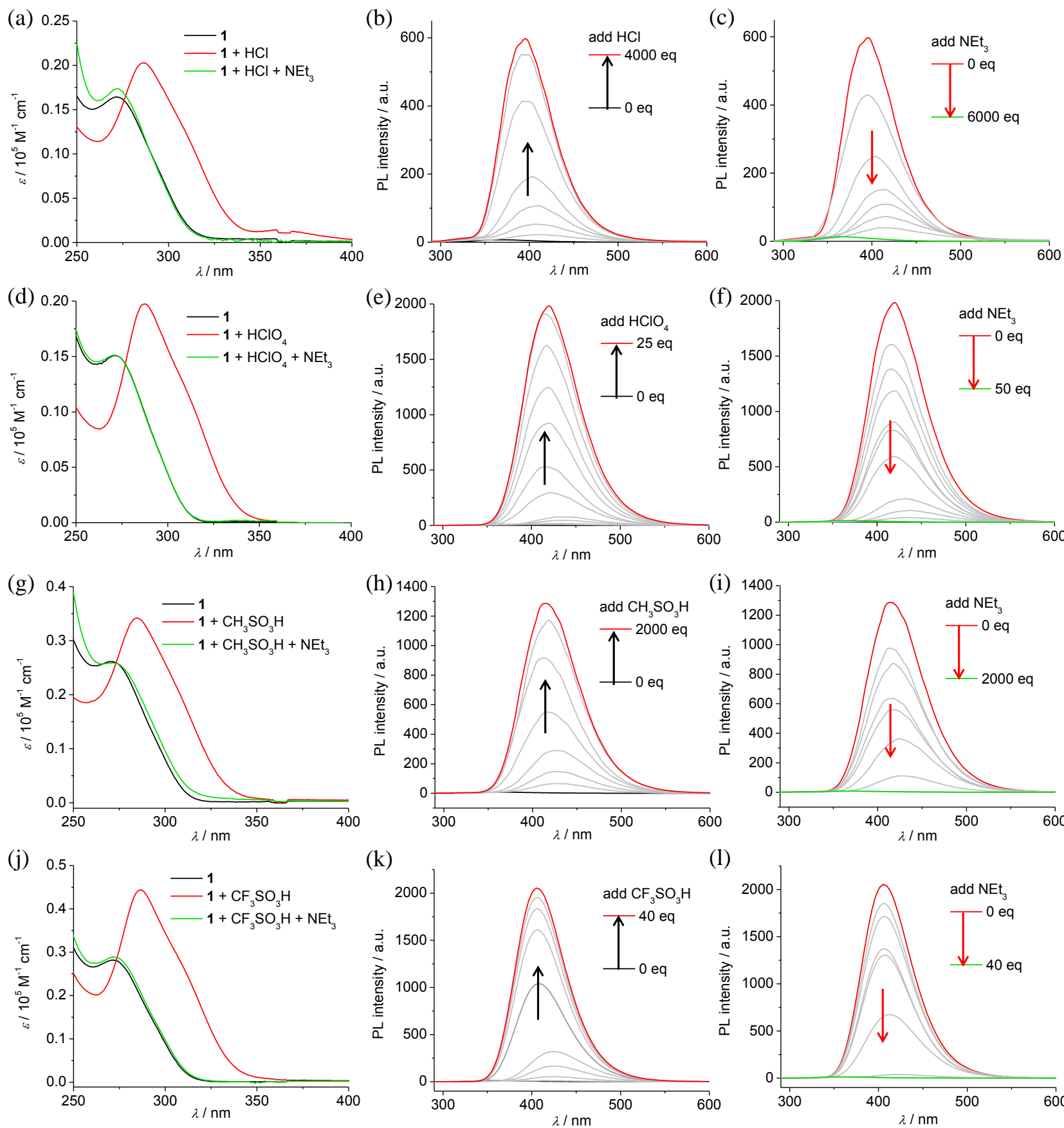

Figure S1. (a, d, g, j) Absorption spectral changes of 1 in THF $\left(5 \times 10^{-5} \mathrm{M}\right)$ upon successive addition of (a) $\mathrm{HCl}$ (200 eq) and $\mathrm{NEt}_{3}$ (600 eq); (d) $\mathrm{HClO}_{4}(2000 \mathrm{eq})$ and $\mathrm{NEt}_{3}(2500 \mathrm{eq})$; (g) $\mathrm{CH}_{3} \mathrm{SO}_{3} \mathrm{H}(2000$ eq) and $\mathrm{NEt}_{3}$ (2000 eq); (j) $\mathrm{CF}_{3} \mathrm{SO}_{3} \mathrm{H}$ (40 eq) and $\mathrm{NEt}_{3}$ (40 eq). (b, c, e, f, h, i, k, l) Emission spectral changes of 1 in THF upon successive addition of different amounts of (b, c) $\mathrm{HCl}$ and $\mathrm{NEt}_{3}$, (e, f) $\mathrm{HClO}_{4}$ and $\mathrm{NEt}_{3}$, (f, h) $\mathrm{CH}_{3} \mathrm{SO}_{3} \mathrm{H}$ and $\mathrm{NEt}_{3},(\mathrm{k}, \mathrm{l}) \mathrm{CF}_{3} \mathrm{SO}_{3} \mathrm{H}$ and $\mathrm{NEt}_{3}$. Excited at $275 \mathrm{~nm}$. 


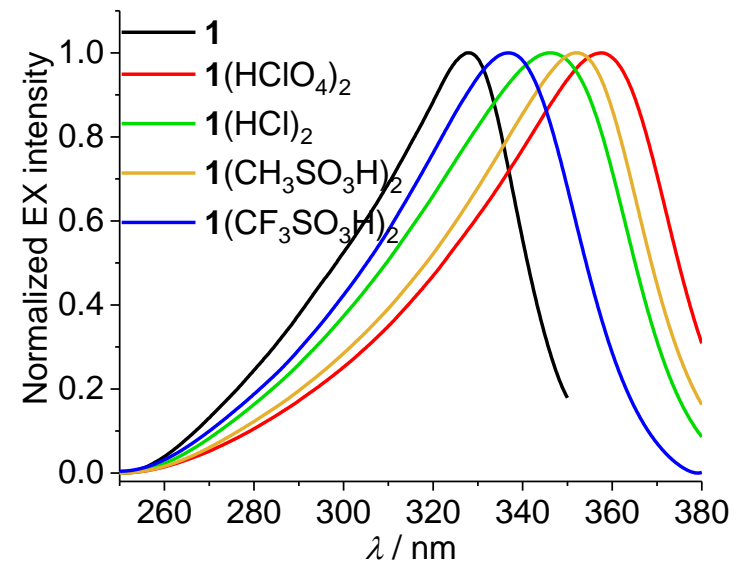

Figure S2. Excitation spectra of crystal 1 and microcrystals $\mathbf{1}(\mathrm{HX})_{2}$. Measured at the maximum emission wavelength.

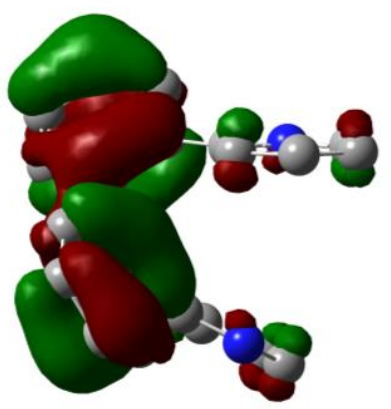

HOMO

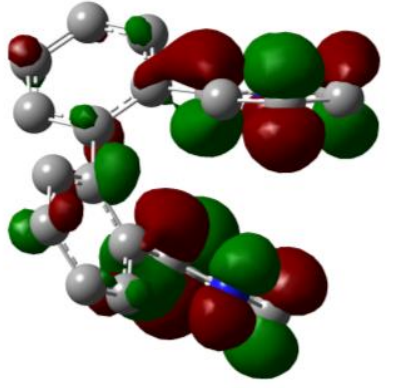

LUMO

Figure S3. HOMO and LUMO of $\left[1 \cdot \mathrm{H}_{2}\right]^{2+}$. Calculated on Gaussian 03 with the B3LYP functional and $6-31 \mathrm{G}^{*}$ basis set. Isovalue $=0.03 \mathrm{e} / \mathrm{bohr}^{3}$. 
(a)

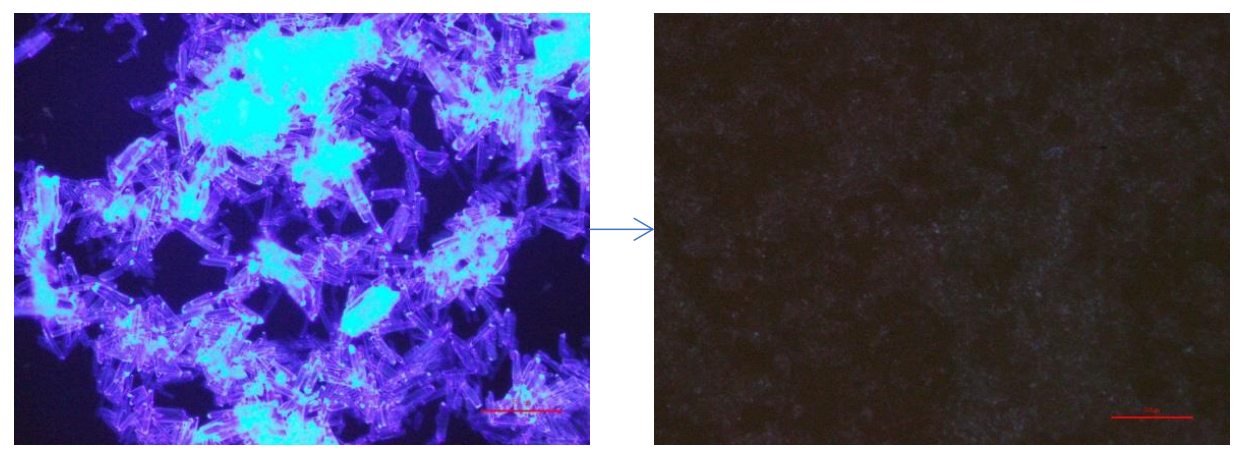

(b)

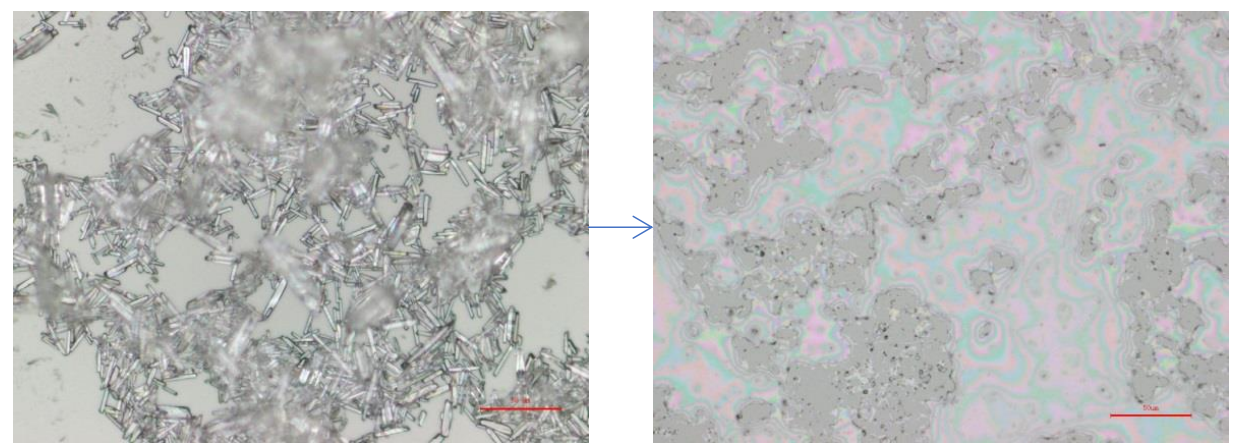

Figure S4. Change of (a) fluorescent microscopic images and (b) optical microscopic images of microcrystals of $\mathbf{1}(\mathrm{HCl})_{2}$ upon heating at $135{ }^{\circ} \mathrm{C}$ for $5 \mathrm{~min}$. 


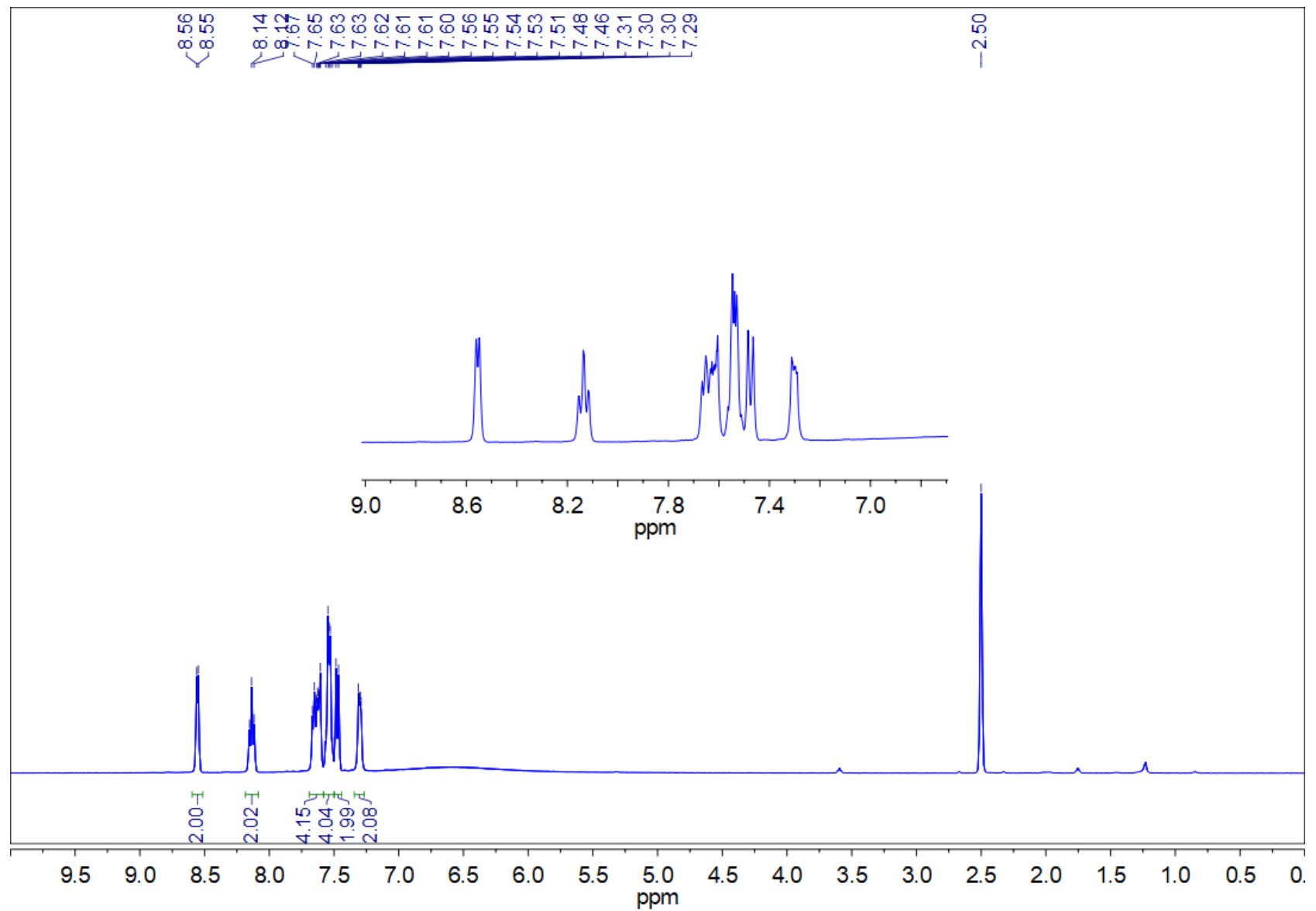

Figure S5. ${ }^{1} \mathrm{H} \mathrm{NMR}$ spectrum of $\mathbf{1}(\mathrm{HCl})_{2}\left(400 \mathrm{MHz}\right.$, in DMSO- $\left.\mathrm{d}_{6}\right)$, the insert shows the enlarged spectrum. 


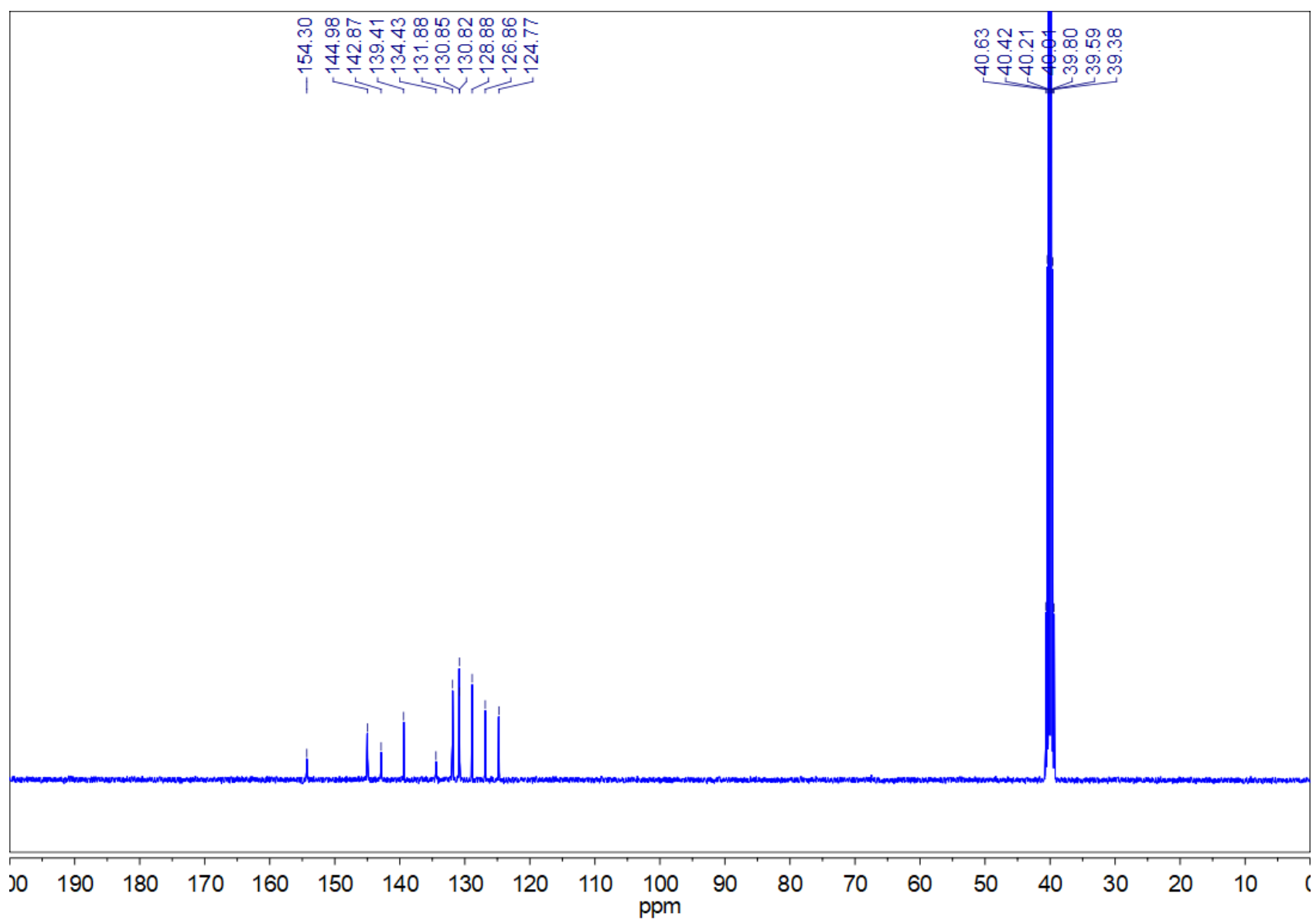

Figure S6. ${ }^{13} \mathrm{C}$ NMR spectrum of $\mathbf{1}(\mathrm{HCl})_{2}\left(400 \mathrm{MHz}\right.$, in DMSO- $\left.\mathrm{d}_{6}\right)$. 


\section{$\mathrm{ESI}(\mathrm{P}), 2 \mathrm{ph}-\mathrm{HCl}, 20201109$}

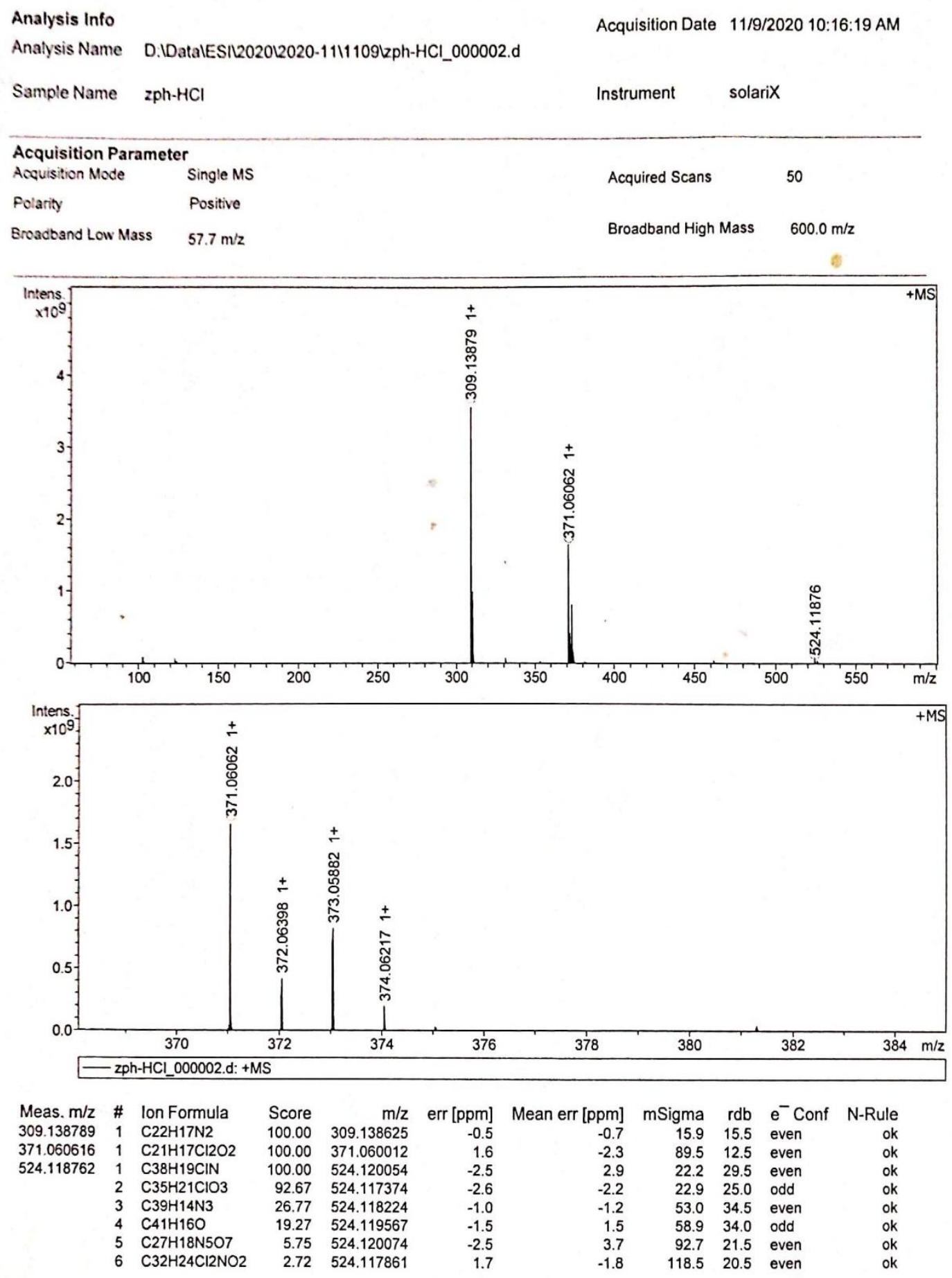

Figure S7. High-resolution ESI(P) mass spectrum of $\mathbf{1}(\mathrm{HCl})_{2}$. 


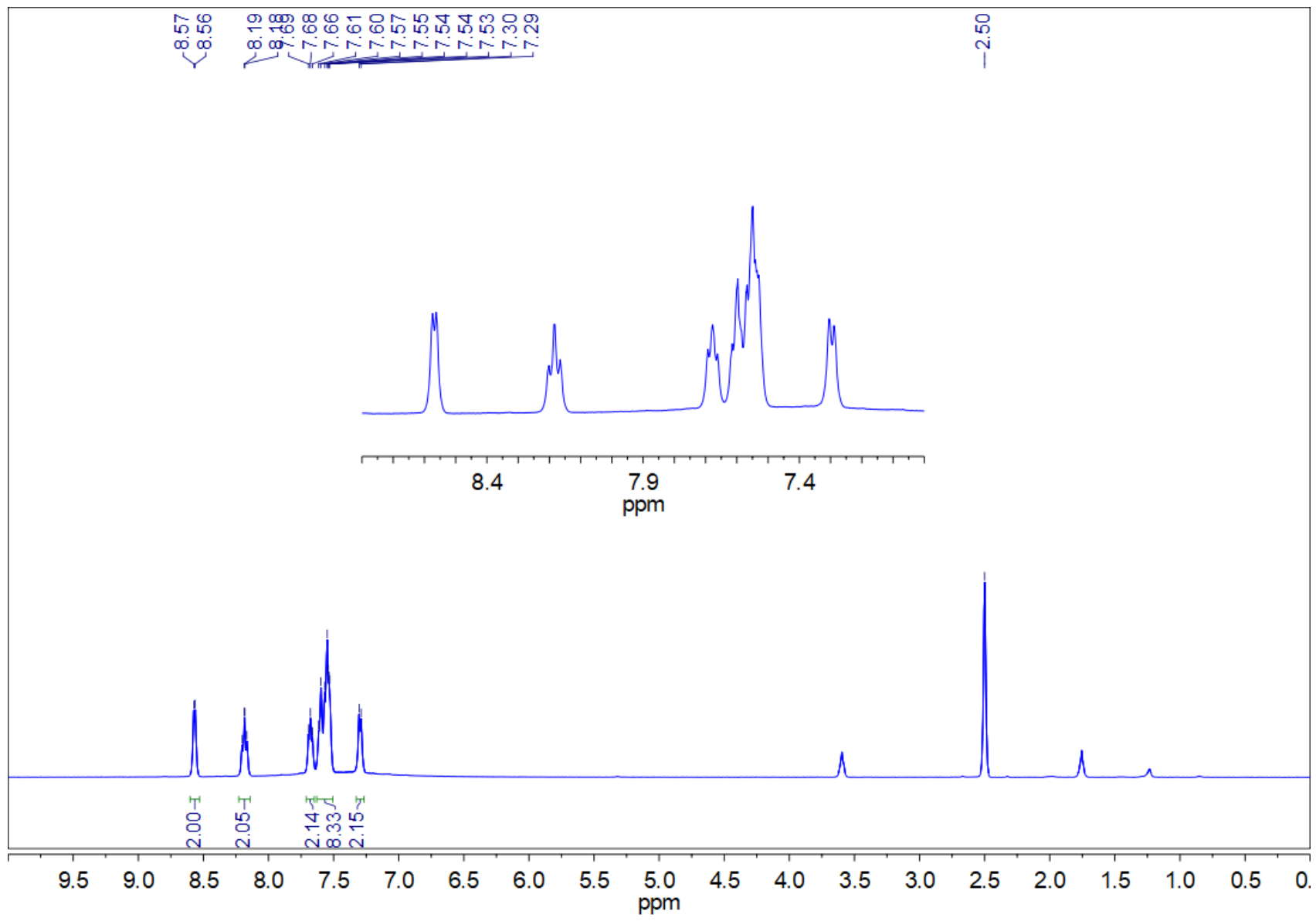

Figure S8. ${ }^{1} \mathrm{H}$ NMR spectrum of $\mathbf{1}\left(\mathrm{HClO}_{4}\right)_{2}$ in $\mathrm{DMSO}-\mathrm{d}_{6}$, the insert shows the enlarged spectrum. 


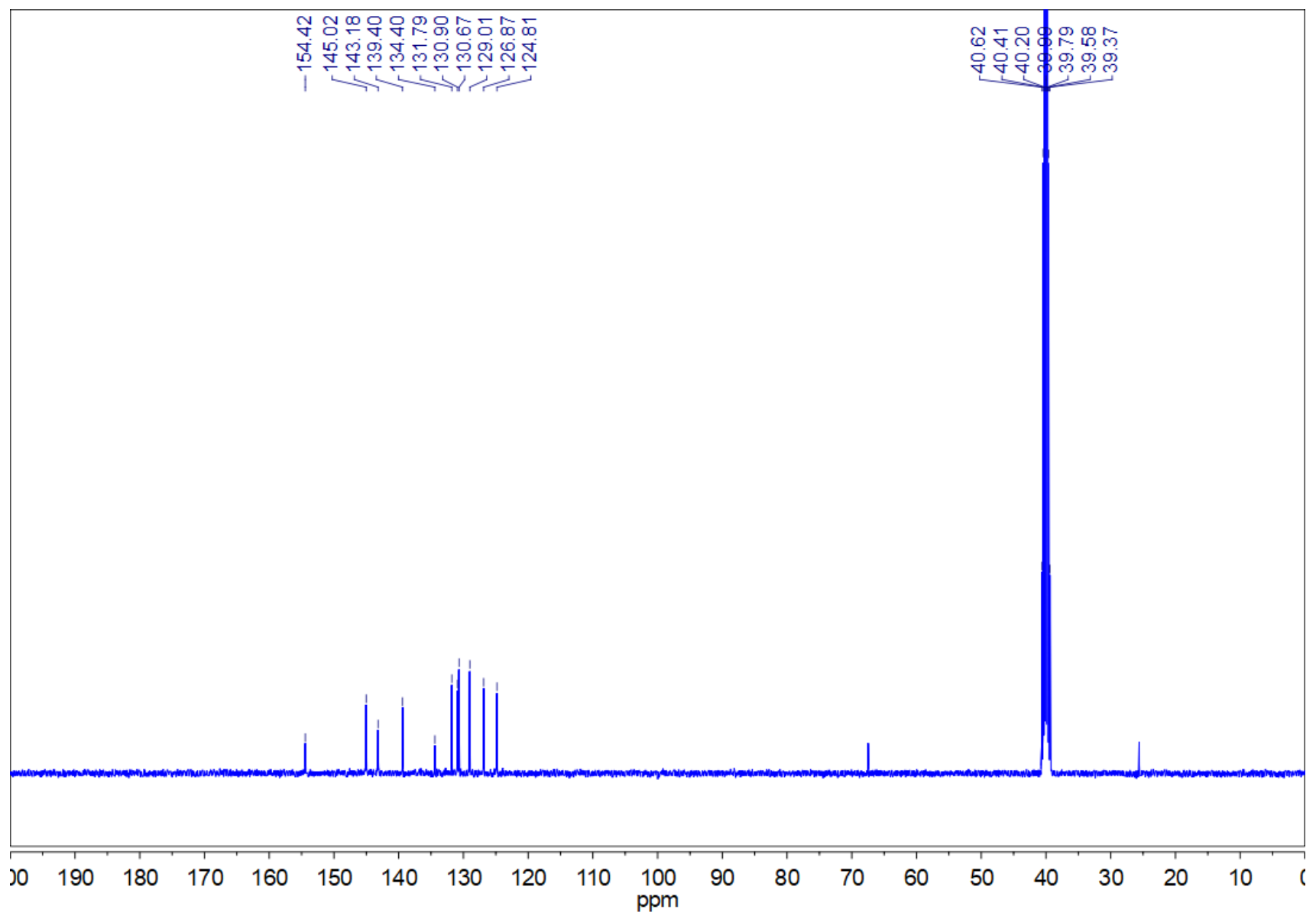

Figure S9. ${ }^{13} \mathrm{C}$ NMR spectrum of $\mathbf{1}\left(\mathrm{HClO}_{4}\right)_{2}$ in DMSO-d $\mathrm{d}_{6}$. 


\section{ESI(P),zph-HClO4,20201109}

Analysis Info

Analysis Name

Sample Name
D:IDatalESIL202012020-1111109lzph-HClO4_000001.d

zph-HClO4
Acquisition Date 11/9/2020 10:25:43 AM

Instrument solariX
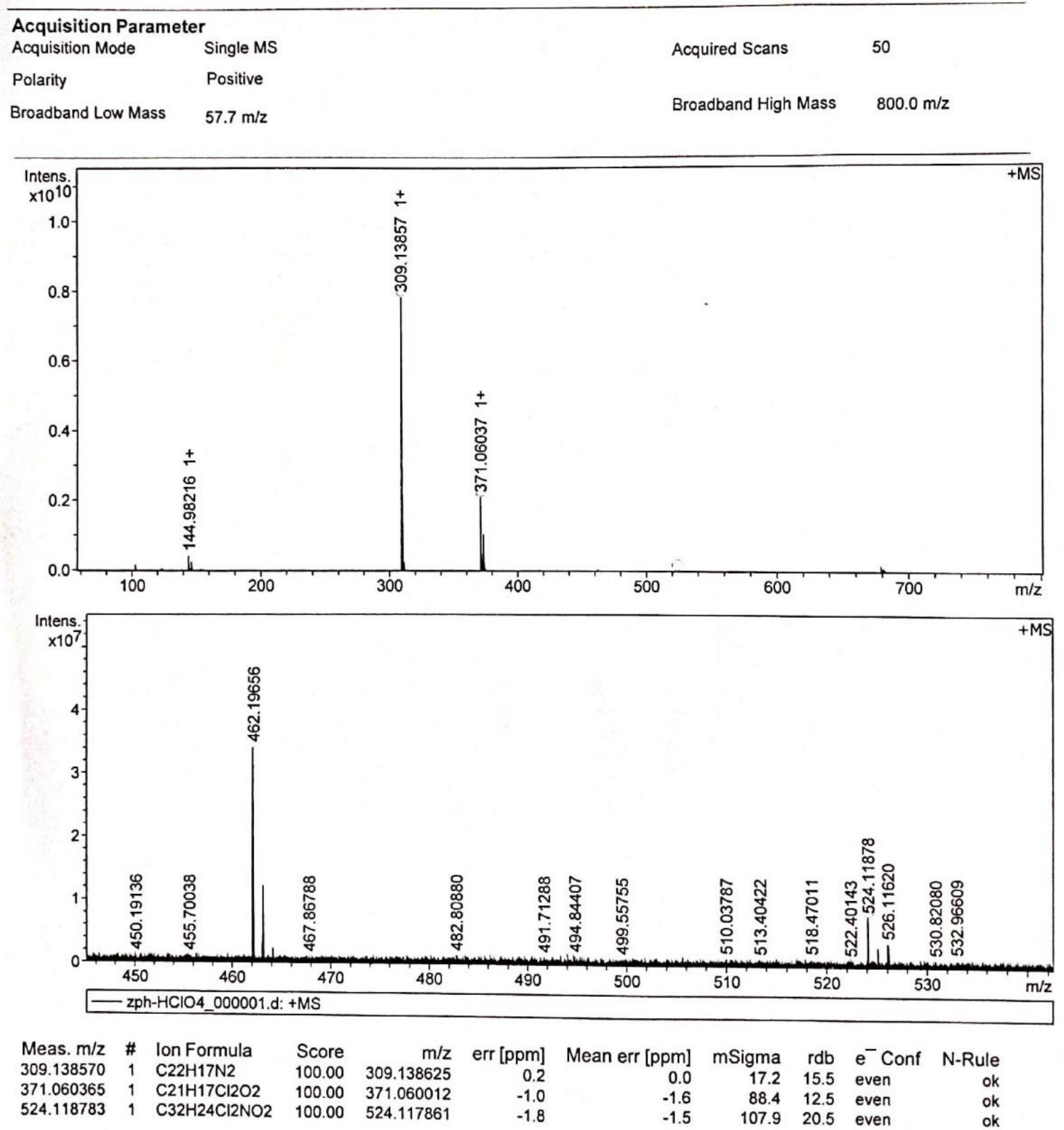

Figure S10. High-resolution ESI $(\mathrm{P})$ mass spectrum of $\mathbf{1}\left(\mathrm{HClO}_{4}\right)_{2}$. 


\section{ESI(N),zph-HClO4,20201109}

Analysis Info

Analysis Name

Sample Name

D:IDatalESII202012020-11/1109lzph-HCIO4_000003.d

zph-HCIO4

Acquisition Parameter

Acquisition Mode Single MS

Polarity

Negative

Broadband Low Mass

$57.7 \mathrm{~m} / \mathrm{z}$
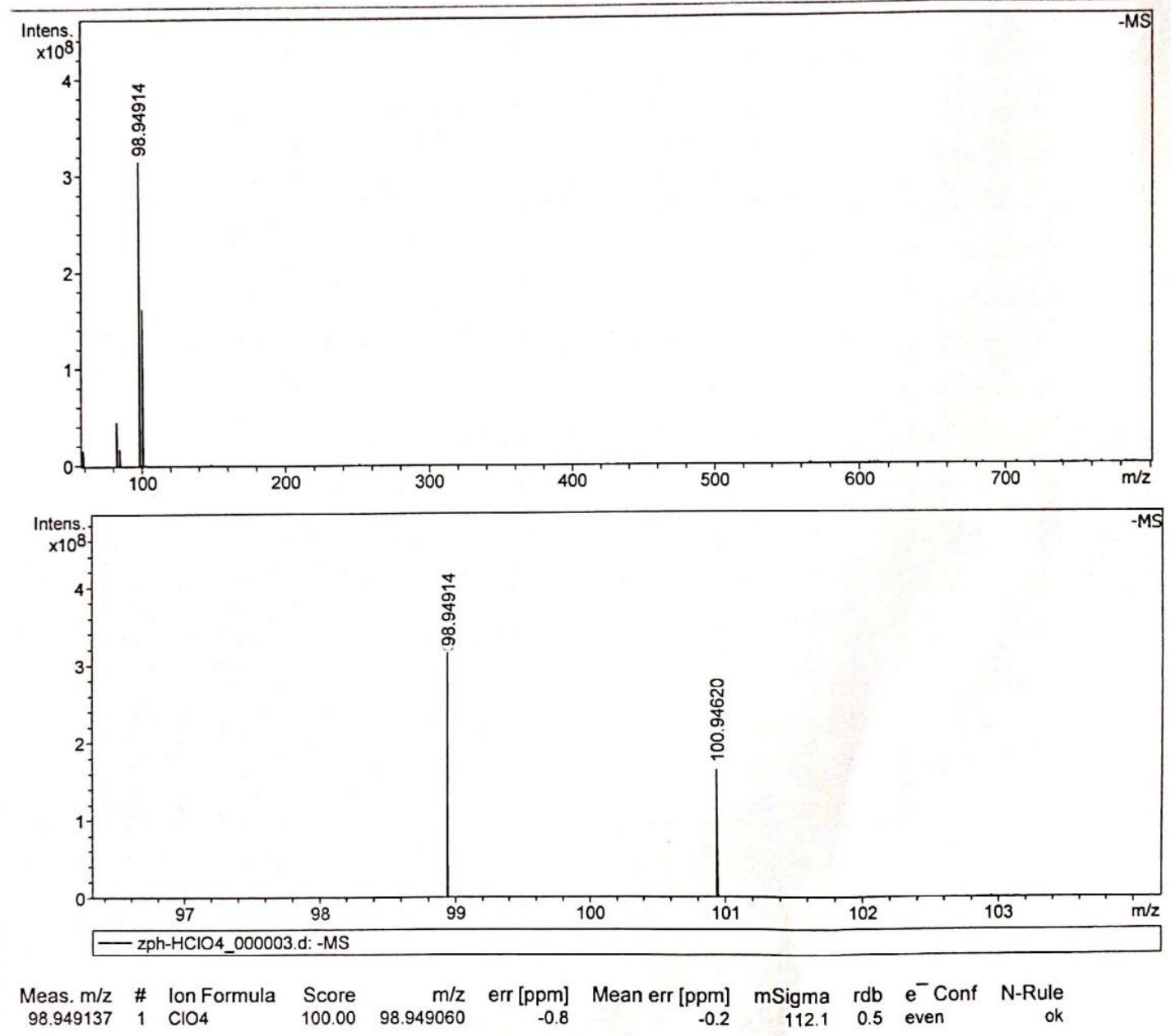

Figure S11. High-resolution ESI(N) mass spectrum of $\mathbf{1}\left(\mathrm{HClO}_{4}\right)_{2}$. 


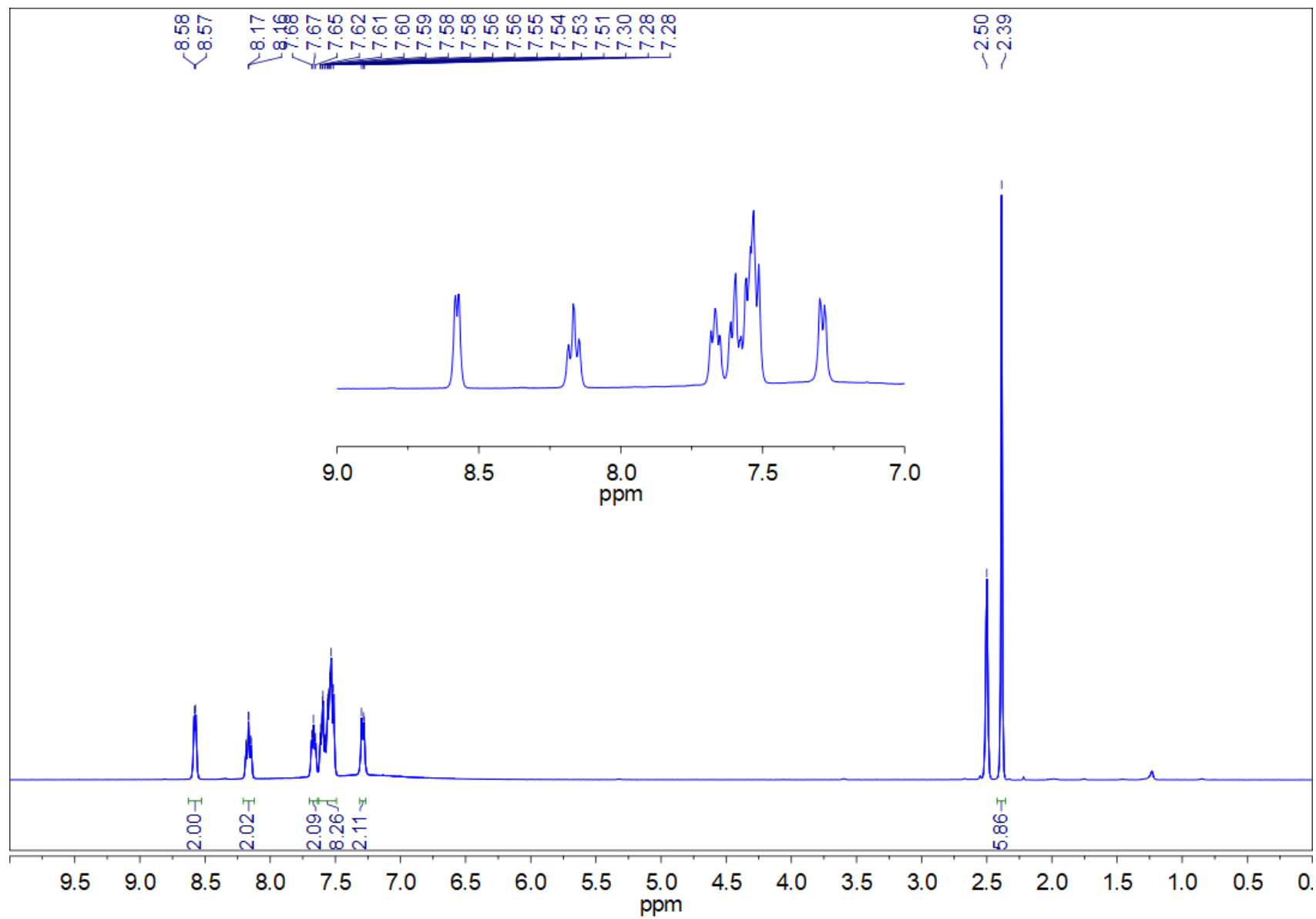

Figure S12. ${ }^{1} \mathrm{H}$ NMR spectrum of $1\left(\mathrm{CH}_{3} \mathrm{SO}_{3} \mathrm{H}\right)_{2}$ in DMSO- $\mathrm{d}_{6}$, the insert shows the enlarged spectrum. 


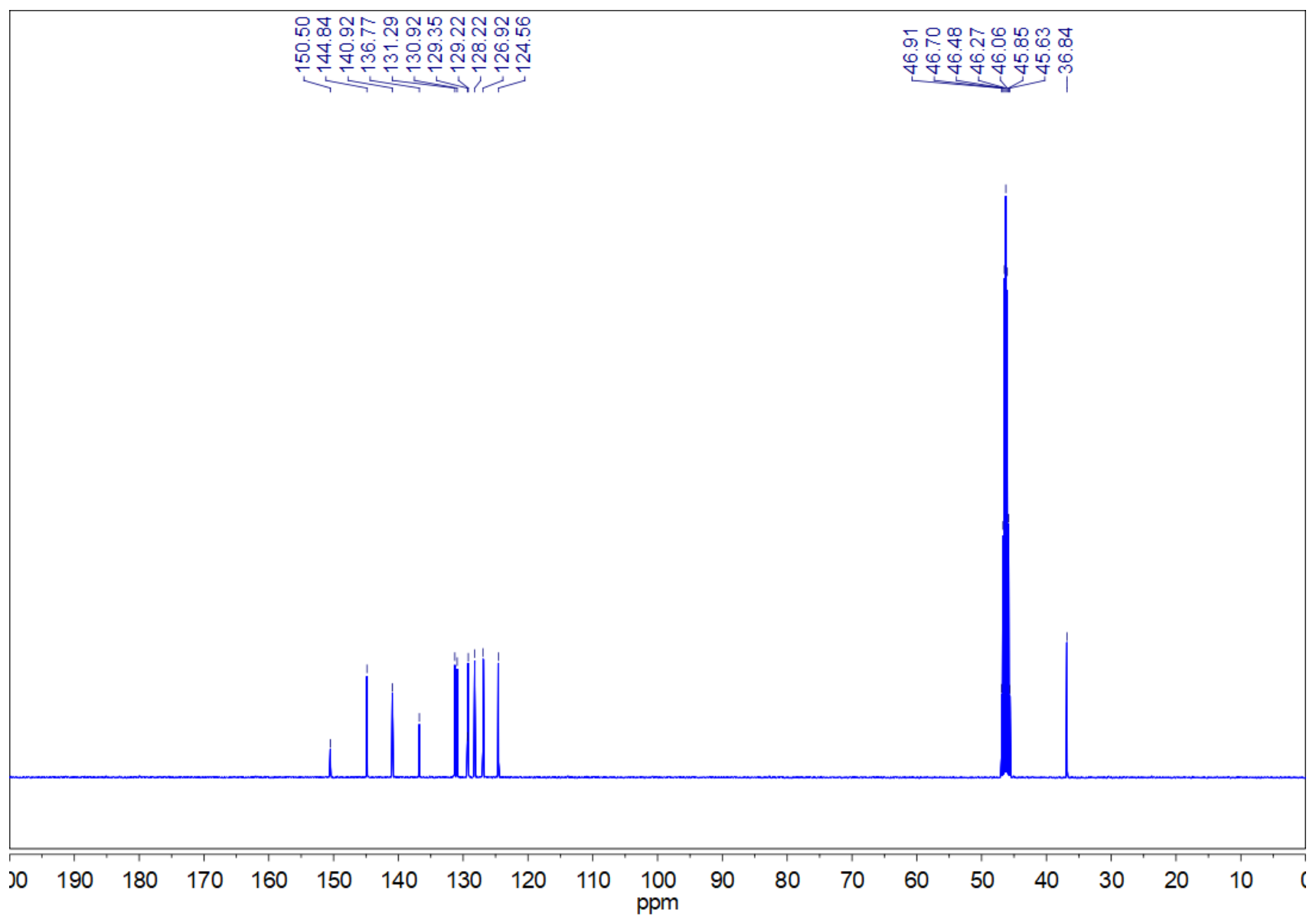

Figure S13. ${ }^{13} \mathrm{C}$ NMR spectrum of $\mathbf{1}\left(\mathrm{CH}_{3} \mathrm{SO}_{3} \mathrm{H}\right)_{2}\left(400 \mathrm{MHz}, \mathrm{CD}_{3} \mathrm{OD}\right)$. 


\section{ESI(P),zph-CH3SO3,20201109}

Analysis Info

Analysis Name

Sample Name

D:IDatalESIL202012020-1111109lzph-CH3SO3_000001.d

zph-CH3SO3

Acquisition Parameter

$\begin{array}{ll}\text { Acquisition Mode } & \text { Single MS } \\ \text { Polarity } & \text { Positive }\end{array}$

Broadband Low Mass

$57.7 \mathrm{~m} / \mathrm{z}$

Acquired Scans

Broadband High Mass $\quad 800.0 \mathrm{~m} / \mathrm{z}$
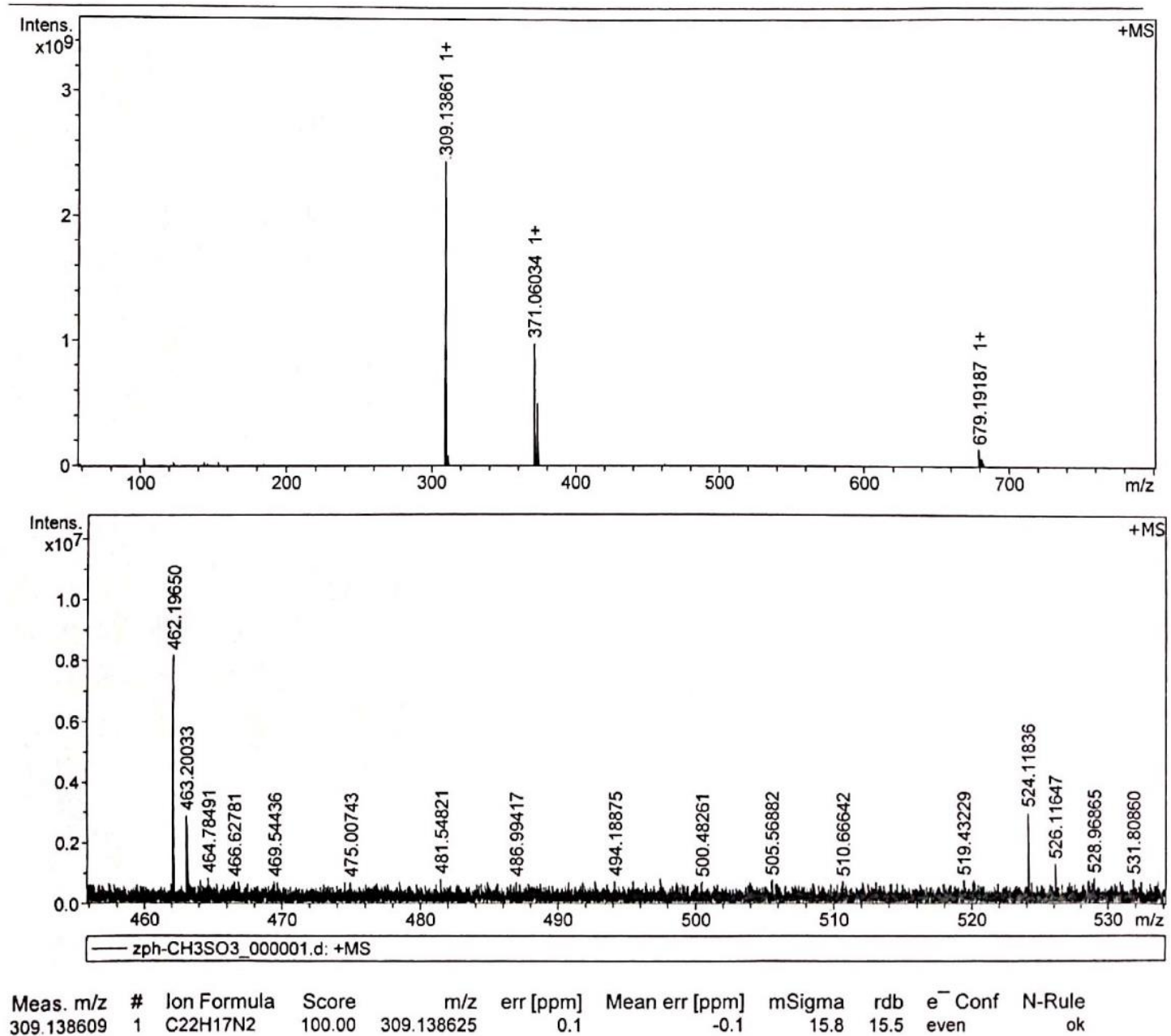

Figure S14. High-resolution ESI $(\mathrm{P})$ mass spectrum of $\mathbf{1}\left(\mathrm{CH}_{3} \mathrm{SO}_{3} \mathrm{H}\right)_{2}$. 


\section{$\mathrm{ESI}(\mathrm{N}), \mathrm{zph}-\mathrm{CH} 3 \mathrm{SO} 3,20201109$}

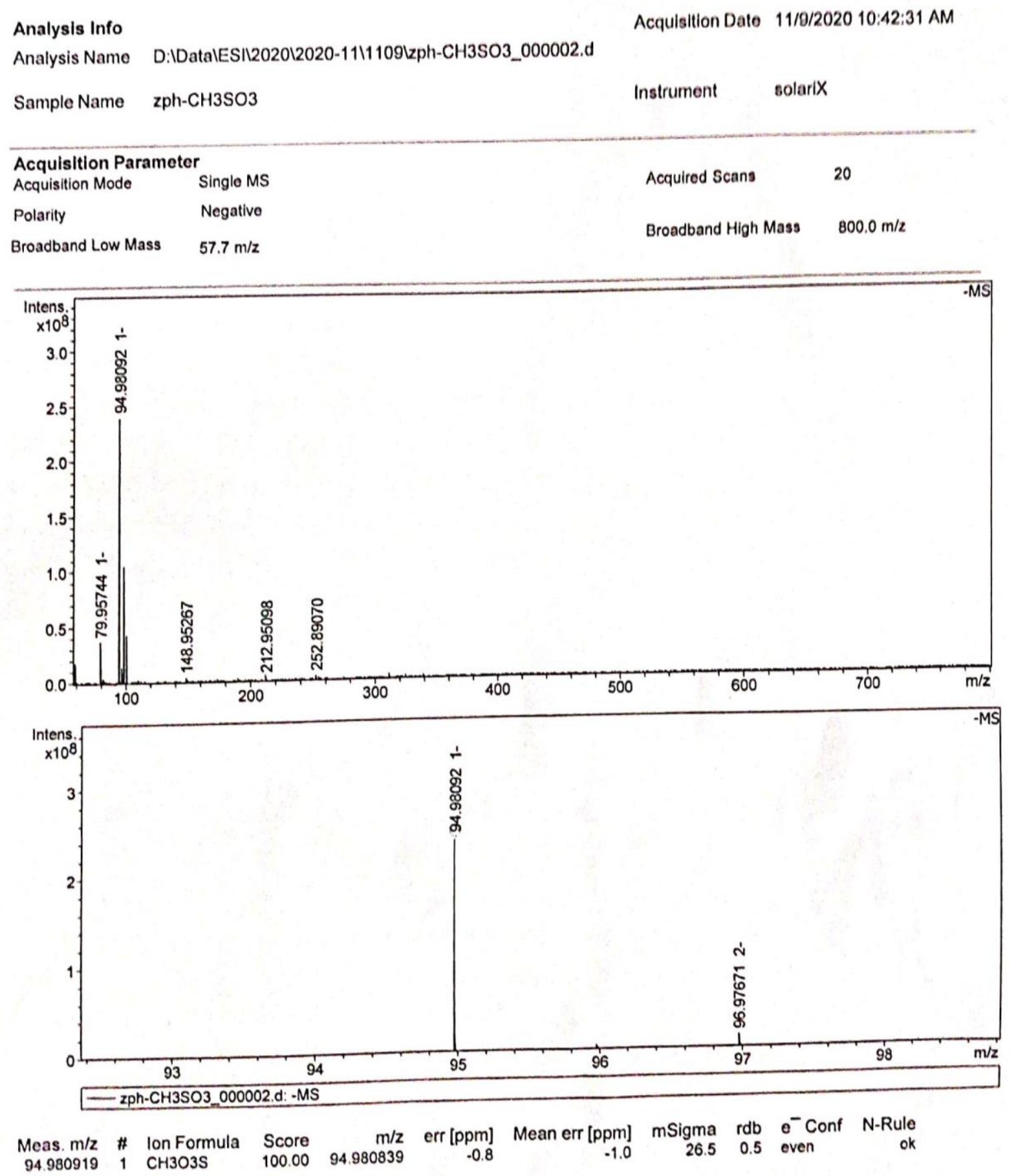

Figure S15. High-resolution ESI(N) mass spectrum of $\mathbf{1}\left(\mathrm{CH}_{3} \mathrm{SO}_{3} \mathrm{H}\right)_{2}$. 


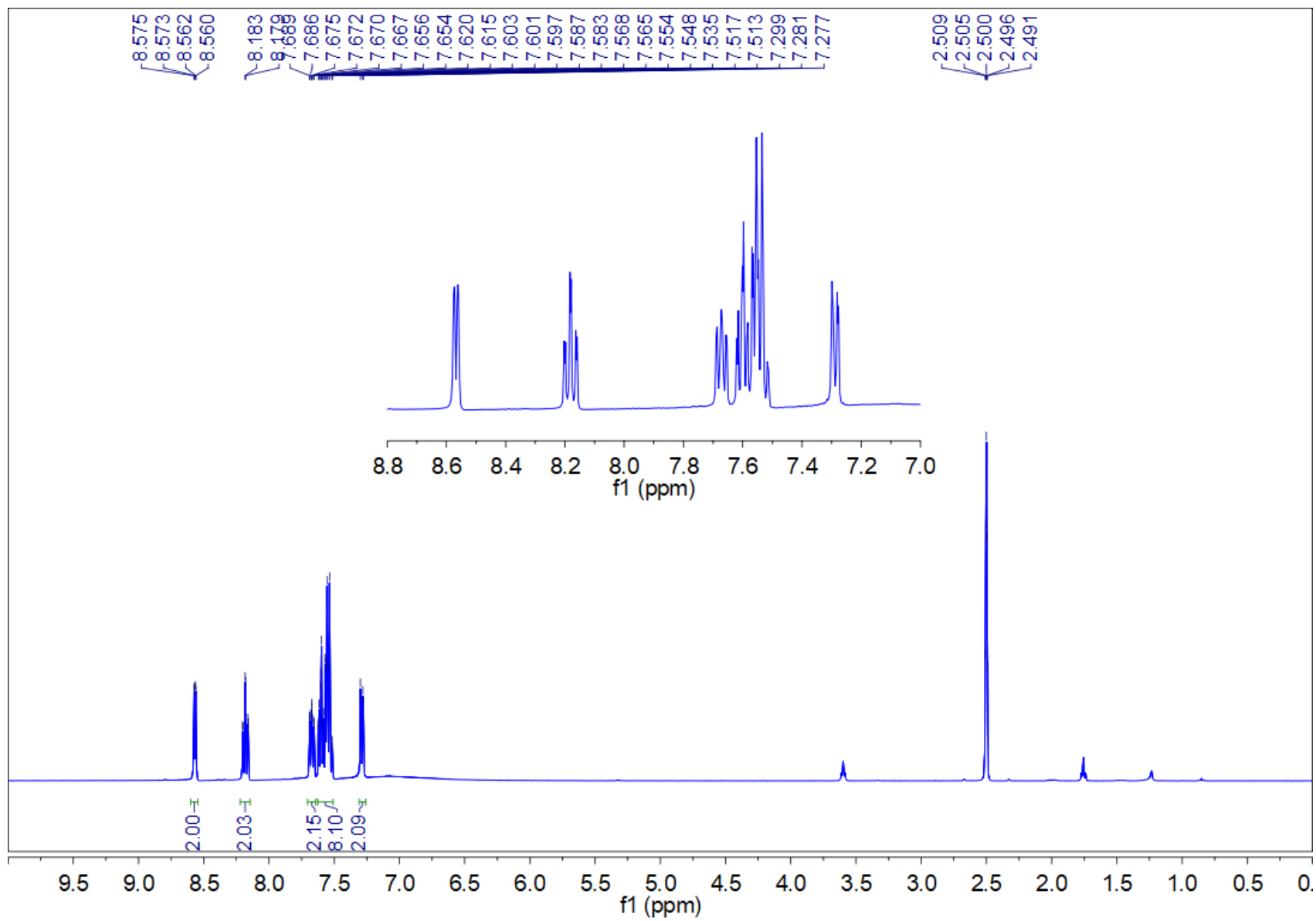

Figure S16. ${ }^{1} \mathrm{H}$ NMR spectrum of $\mathbf{1}\left(\mathrm{CF}_{3} \mathrm{SO}_{3} \mathrm{H}\right)_{2}$ in DMSO- $\mathrm{d}_{6}$, the insert shows the enlarged spectrum. 


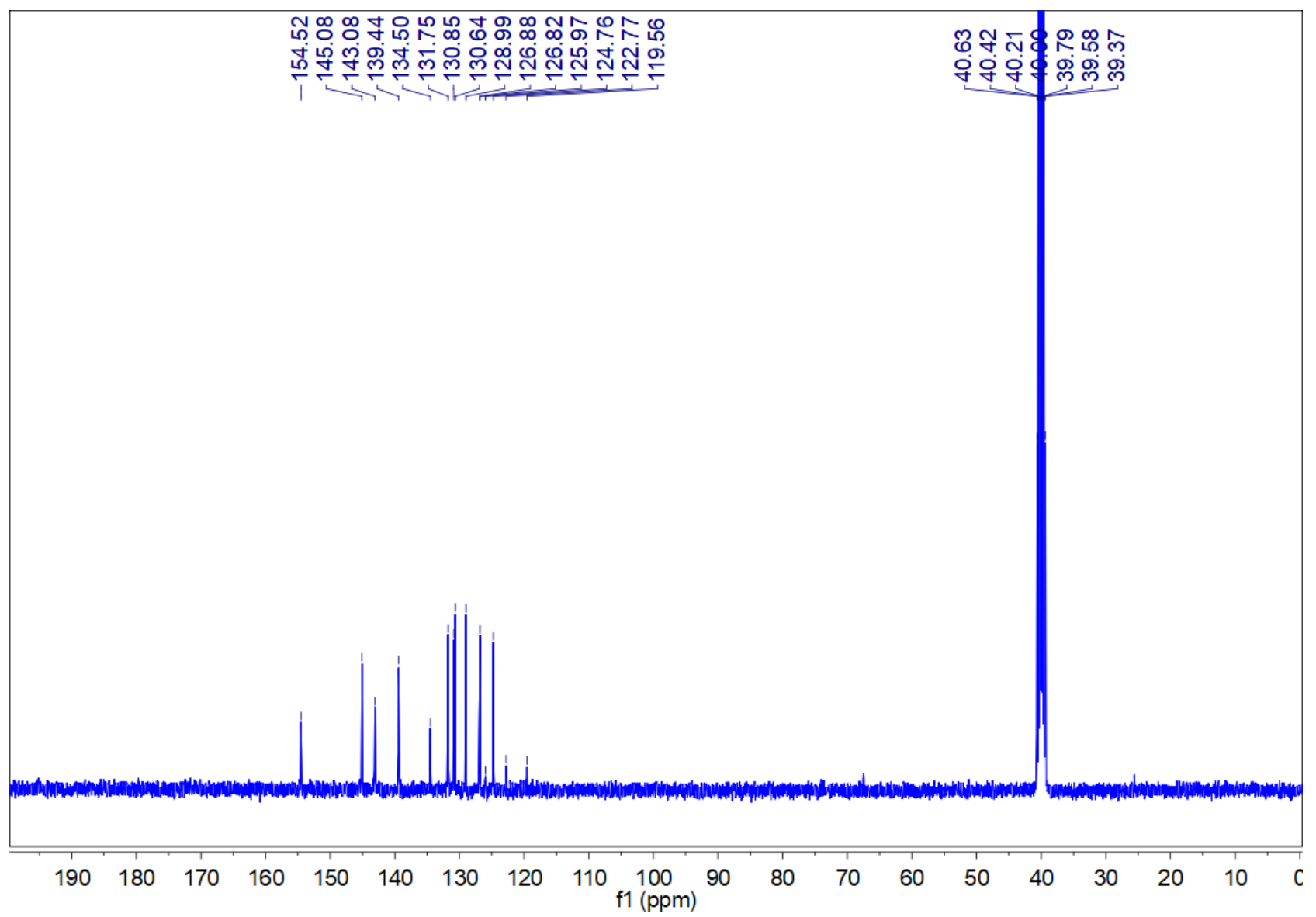

Figure S17. ${ }^{13} \mathrm{C}$ NMR spectrum of $1\left(\mathrm{CF}_{3} \mathrm{SO}_{3} \mathrm{H}\right)_{2}$ in DMSO-d $\mathrm{d}_{6}$. 


\section{ESI(P),zph-CF3SO3,20201109}

Analysis Info

Analysis Name D:IDatalESIL202012020-1111109lzph-CF3SO3_000002.d

Sample Name zph-CF3SO3
Acquisition Date 11/9/2020 10:48:34 AM

Instrument solariX
Acquisition Parameter

Acquisition Mode Single MS

Polarity Positive

Broadband Low Mass

$57.7 \mathrm{~m} / \mathrm{z}$
10

Acquired Scans

Broadband High Mass $\quad 800.0 \mathrm{~m} / \mathrm{z}$
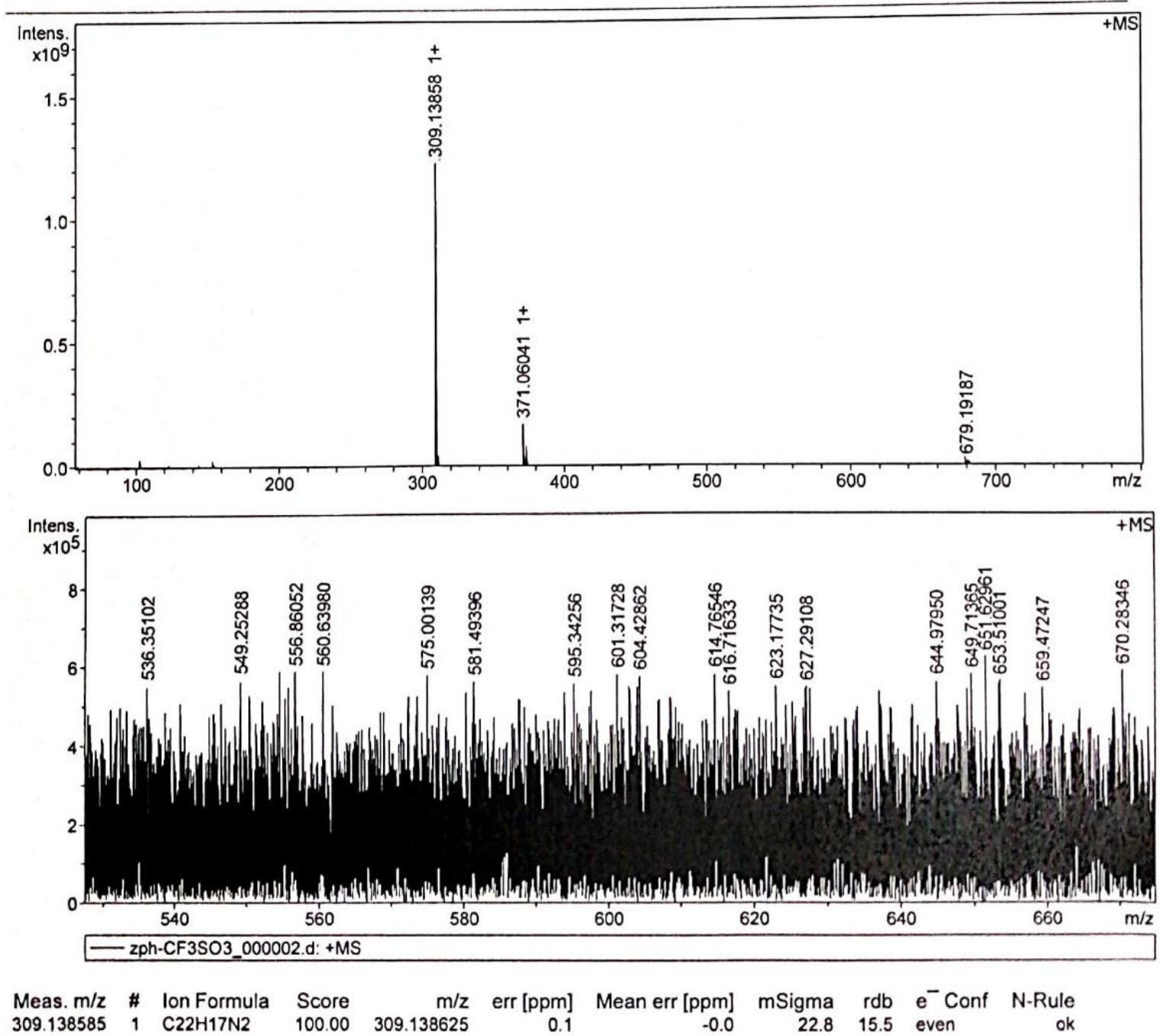

Figure S18. High-resolution ESI $(\mathrm{P})$ mass spectrum of $\mathbf{1}\left(\mathrm{CF}_{3} \mathrm{SO}_{3} \mathrm{H}\right)_{2}$. 


\section{ESI(N),zph-CF3SO3,20201109}

Analysis Info

Analysis Nam

Samplo Name
D:IDataIESIL202012020-11111091zph-CF3SO3_000001.d

zph-CF3SO3

Acquisition Date 11/9/2020 10:48:00 AM

Instrument

solariX
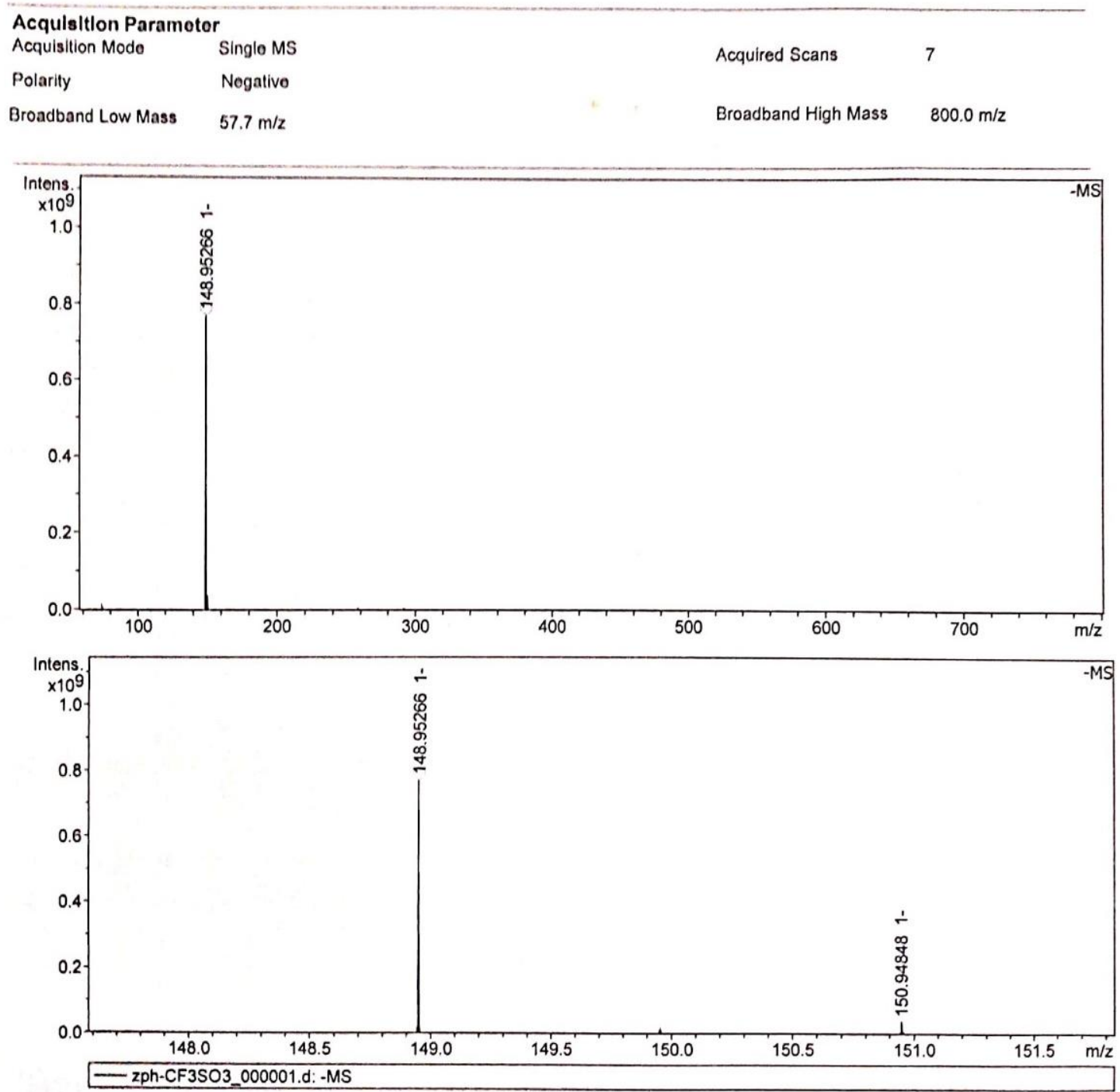

Meas. $\mathrm{m} / \mathbf{z}$ \# Ion Formula Score $\mathrm{m} / \mathrm{z}$ err [ppm] Mean err [ppm] mSigma rdb $\mathrm{e}^{-}$Conf $\mathrm{N}-\mathrm{Rule}$

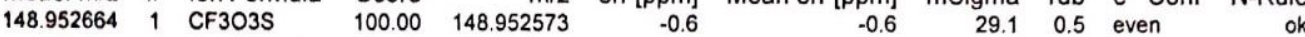

Figure S19. High-resolution ESI(N) mass spectrum of $\mathbf{1}\left(\mathrm{CF}_{3} \mathrm{SO}_{3} \mathrm{H}\right)_{2}$. 